\title{
Inclusion of Real-Time In-Situ Measurements into the UCSD Time-Dependent Tomography and Its Use as a Forecast Algorithm
}

\author{
B.V. Jackson · J.M. Clover · P.P. Hick • A. Buffington • \\ M.M. Bisi • M. Tokumaru
}

Received: 2 February 2012 / Accepted: 8 August 2012 / Published online: 27 September 2012 (C) The Author(s) 2012. This article is published with open access at Springerlink.com

\begin{abstract}
The University of California, San Diego (UCSD) three-dimensional (3D) timedependent tomography program, used for over a decade to reconstruct and forecast coronal mass ejections (CMEs), does so from observations of interplanetary scintillation (IPS) taken using the Solar-Terrestrial Environment Laboratory (STELab) radio arrays in Japan. An earlier article (Jackson et al. in Solar Phys. 265, 245, 2010) demonstrated how in-situ velocity measurements from the Advanced Composition Explorer (ACE) space-borne instrumenta-
\end{abstract}

Observations and Modelling of the Inner Heliosphere

Guest Editors: Mario M. Bisi, Richard A. Harrison, and Noé Lugaz

B.V. Jackson $(\bowtie) \cdot$ J.M. Clover · P.P. Hick · A. Buffington · M.M. Bisi

Center for Astrophysics and Space Sciences, University of California, San Diego, 9500 Gilman

Drive \#0424, La Jolla, 92093-0424 CA, USA

e-mail: bvjackson@ucsd.edu

J.M. Clover

e-mail: jclover@ucsd.edu

P.P. Hick

e-mail: pphick@ucsd.edu

A. Buffington

e-mail: abuffington@ucsd.edu

P.P. Hick

San Diego Supercomputer Center, University of California, San Diego, 9500 Gilman Drive \#0505, La Jolla, 92093-0505 CA, USA

e-mail: pphick@ucsd.edu

M.M. Bisi

Institute of Mathematical and Physical Sciences, Aberystwyth University, Penglais Campus, Aberystwyth, SY23 3BZ Ceredigion, Wales, UK

e-mail: Mario.Bisi@aber.ac.uk

M. Tokumaru

Solar-Terrestrial Environment Laboratory (STELab), University of Nagoya, Furo-cho, Chikusa-ku, Nagoya 464-8601, Japan

e-mail: tokumaru@stelab.nagoya-u.ac.jp 
tion can be used in addition to remote-sensing data to constrain a time-dependent tomographic velocity solution. Here we extend this in-situ inclusion to density measurements, and show how this constrains the tomographic density solution. Supplementing remote-sensing observations with in-situ measurements provides additional information to construct an iterated solar-wind parameter that is propagated outward from near the solar surface past the measurement location, and throughout the volume. As in the case of velocity when this is done, the largest changes within the volume are close to the radial directions around Earth that incorporate the in-situ measurements; the inclusion significantly reduces the uncertainty in extending these measurements to global 3D reconstructions that are distant in time and space from the spacecraft. At Earth, this analysis provides a finely tuned real-time result up to the latest time for which in-situ measurements are available, and enables more-accurate extension of these results near Earth to those remotely sensed. We show examples of this new algorithm using real-time STELab IPS data that were used in our forecasts throughout Carrington rotations 2010 through 2016, and we provide one metric prescription that we have used to determine the forecasting accuracy one, two, and three days in advance of the time data become available to analyze from STELab. We show that the accuracy is considerably better than assuming persistence of the same signal over one to two days in advance of when the data are available.

\section{Introduction}

Observations of interplanetary scintillation (IPS) of meter-wavelength intensity variations from point radio sources have long been a source of heliospheric remote-sensing information. IPS measures small-scale $(\sim 150 \mathrm{~km})$ density variations along the line of sight (LOS) to a radio source (e.g., Hewish, Scott, and Wills, 1964; Ananthakrishnan, Coles, and Kaufman, 1980).

In Jackson et al. (2010a) (hereafter Paper 1) we describe some of the background analyses that have been used at the University of California, San Diego (UCSD) to provide threedimensional (3D) depictions of heliospheric structures using IPS techniques. The reader is asked to refer to this article (and references therein) for more details introducing these analyses. Over the years a Computer Assisted Tomography (CAT) program has been developed (Jackson et al., 1998, 2003, 2010d; Kojima et al., 1998; Jackson, Hick, and Buffington, 2002; Hick and Jackson, 2004; Jackson and Hick, 2005) that iteratively fits remotely-sensed observations to a kinematic solar-wind model. Other articles detail how the time-dependent tomographic analyses can be used to map to velocities and densities to near Earth (e.g., Jackson et al., 2006, 2008, 2010c; Bisi et al., 2007, 2009a, 2010a), at Mars (Jackson et al., 2007), and at the Solar TErrestrial RElations Observatory (STEREO) spacecraft (Kaiser et al., 2008). See Bisi et al. (2009b) and Jackson et al. (2011), for the STEREO analyses. This technique has performed well in retrospective analyses using a variety of remote-sensing data sets.

Using our tomographic technique we have fit data from the Solar-Terrestrial Environment Laboratory (STELab; Nagoya University, Japan) (Kojima and Kakinuma, 1987), and in the past operated a real-time forecasting system during the nine-month period each year when the STELab IPS arrays were operational. From early in the last decade up until the beginning of 2011, these IPS results were compared with velocities and densities measured in real time available from the Advanced Composition Explorer (ACE) (Stone et al., 1998) Solar Wind Electron Proton Alpha Monitor (SWEPAM) (McComas et al., 1998) instrumentation. In the spring of 2011, the time-dependent forecasts at UCSD have been carried out in a way that is different from the original technique. 
Forecasts of density and velocity prior to 2011 have often not been as accurate as possible, and in Paper 1 we describe a way to rectify this by including available in-situ velocity information near the Earth into the forecast. This extension more accurately reproduces the ACE in-situ velocity measurements at Earth at the appropriate spatial and temporal resolutions of the 3D reconstructions. It also shows that with this extension the global tomographic solution is not significantly affected. Remaining differences between the in-situ densities and velocities at Earth and their remotely-sensed counterpart can provide insight into the 3D structures of which they are a part, and can also be used to more accurately extend spacecraft in-situ measurements to locations only accessed remotely. Since the spring of 2011, we have extended our analyses to include in-situ measurements of density as well as velocity, and in this paper we describe how this density inclusion is accomplished with the STELab IPS data. Unlike the analysis shown in Paper 1 both in-situ velocities and densities are used to provide accurate weighting along each near-Earth LOS portion. This new technique provides a match of velocity and now density at Earth superior to the former technique. In this paper, we provide a metric that describes the extent to which this new technique can be judged to fit the ACE in-situ velocities and densities that are also compared with the remotely-sensed forecast values.

Section 2 describes the tomographic program developed to fit IPS data that has been extended to now include in-situ density measurements. Section 3 compares results for a portion of Carrington rotation (CR) using a combination of remote-sensing observations and in-situ density and velocity measurements. Section 4 provides a forecast metric that is used to determine how well the forecast works when including available in-situ density and velocity information. Section 5 presents a summary and conclusions.

\section{3D Reconstructions Using In-Situ Density Measurements}

The UCSD remote-sensing reconstruction technique provides 3D solar-wind density and velocity by applying an inversion technique to the IPS data. For a more complete description of the time-dependent analysis using either IPS or Thomson-scattering data see Jackson et al. (2010d) and references therein. When a transient structure such as a heliospheric response to a coronal mass ejection (CME) crosses a large range of solar elongations, it is viewed from widely different directions. Both this changing perspective and the changing LOS weighting for the outwardly expanding solar-wind material are exploited to reconstruct a 3D timedependent solar-wind model, as explained more fully in Paper 1.

In addition to its use at UCSD this same time-dependent analysis system is extant at the NASA Goddard Community Coordinated Modeling Center (CCMC) for utilizing STELab IPS data. This analysis maps the heliosphere using all available data covering an interesting period of observation, including data both prior to and well after the ejected solar material moves past $1 \mathrm{AU}$. These compare favorably with other techniques used to invert the IPS data to show the extent of CME structures (e.g., Tokumaru et al., 2005, 2007) using archival data sets. The program can be operated in two different modes. The second mode of operation of the UCSD program uses data up to the present, and allows a forecast of the arrival of transient heliospheric structures. Since the summer of 2011 this analysis system now also provides an option to include in-situ measurements of velocity and density as described in this article.

For the analyses and comparisons presented here, we include ACE Level-0 hourly averaged data since these are readily available in real time. For a discussion of the inclusion of ACE velocity data into the time-dependent tomography the reader is referred to Paper 1. 
Figure 1 IPS $327 \mathrm{MHz}$ LOS

weighting with distance from Earth as described by Jackson $e t$ al. (1998). The arrow gives the location of the weighting superseded by the in-situ measurement.

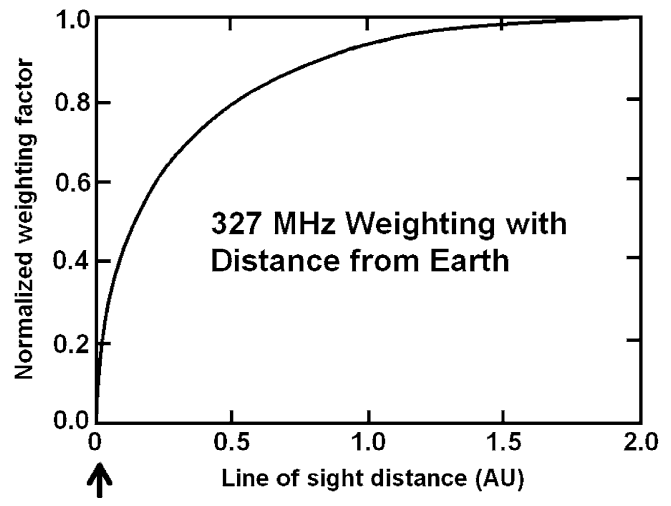

The remotely-sensed STELab data from the new Toyokawa Solar Wind Imaging Facility Telescope (SWIFT) are currently used exclusively for the $g$-level (normalized scintillation index) IPS analyses (Tokumaru et al., 2011). The $g$-level is related to the scintillation index $m$ by

$$
g=m /\langle m\rangle,
$$

where $m$ is the instantaneous observed scintillation index for an IPS source, and $\langle m\rangle$ is the expected "quiet" scintillation index based on an average of past source observations as a function of solar elongation. The solar-wind model provides the density, and this is converted to the scintillation level by a two-parameter process that is used to fit general solar-wind conditions at the Earth. The scintillation index $m$ is related to small-scale density variations along the line of sight $s$ by

$$
m^{2}=\int_{0}^{\infty} \mathrm{d} s W \rho(s) \delta n(s)^{2} .
$$

The "weight function" $W \rho(s)$ as formulated by Young (1971) and refined by Kojima et al. (1998), depends upon the observing wavelength and the angular size of the radio source. This weighting is shown at $327 \mathrm{MHz}$ for a source size of 0.1 arcsec in Figure 1. It is this portion of the curve that varies significantly for different source sizes from the standard 0.1 $\operatorname{arcsec}$ (see Bisi et al., 2010b). The same weak-scattering weight function is assumed for fast and slow solar wind and differing solar-wind structure. The small-scale density fluctuations $\delta n(s)$ along the line of sight do not only depend on macroscopic properties (solar-wind speed, density, magnetic field), but also on microscopic properties associated with turbulence in the solar wind. However, empirical evidence suggests that changes in $\delta n(s)$ scale with changes in values of the bulk electron density. Quantitatively, we model this behavior (see Jackson et al., 1998, 2010d) by expressing $\delta n(s)$ in terms of the heliocentric distance $r$ and the solar-wind electron density $n$, or, equivalently, the normalized density defined as $\hat{n}=\left(r / r_{0}\right)^{2} n$ :

$$
\delta n(r, \hat{n})=\delta n_{0}\left(r_{0} / r\right)^{2-\beta r}\left(\hat{n} / n_{0}\right)^{\beta n} .
$$

A value for $r_{0}$ of $1 \mathrm{AU}$ normalizes results to observations at Earth's distance from the Sun (Kojima et al., 1998). The tomography is calculated using values for the powers $\beta n$ and $\beta r$ that are derived by iteratively fitting remote-sensing results to in-situ solar-wind velocity and density measurements (Jackson et al., 1998, 2003). Physically, the value of $\beta n$ relates 
the small-scale scattering level to bulk density at $1 \mathrm{AU}$ while the value of $\beta r$ provides for a change in this relationship with solar distance. This relationship is explored more fully in Jackson et al. (1998) and Asai et al. (1998). For the new Toyokawa array, the two parameters $\beta n=0.40$ and $\beta r=0$ were set using an iterative procedure that determined the best fit for proton density number $\mathrm{cm}^{-3}(\mathrm{~Np})$ by iteratively altering these parameters for a period in early 2011 (CR2105-CR2106) to best match ACE Level-0 data. For use in the analysis, we note that for conversion of in-situ density near Earth to $g$-level only the latter term of Equation (3) that utilizes the power $\beta n=0.40$ is employed. Although these two parameter fits have been used throughout 2011 to match the IPS tomography to ACE data fairly well, both of these parameters, but especially $\beta n$, can vary with ongoing radio array calibration, or for that matter different in-situ instrument data reduction techniques or plasma instruments. Thus, it is expected that these may need revision on an annual basis or when the Toyokawa IPS array is re-calibrated, or for use with other in-situ data sets such as that from the Wind spacecraft (Ogilvie and Desch, 1997), or the Charge, Element, Isotope Analysis System (CELIAS) Proton Monitor (Hovestadt et al., 1995) on board the SOlar and Heliospheric Observatory (SOHO) (Domingo, Fleck, and Poland, 1995).

The in-situ measurements are integrated into the reconstruction as a "line of sight" with a single LOS segment close to Earth. Because global density measurements strongly depend on the LOS scintillation weighting, an alternate method such as the in-situ inclusion that provides a proper analysis at this LOS location is of high significance. The in-situ inclusion supersedes this weighting by imposing more significance on the in-situ value (at the location of the arrow in Figure 1). Weighting of in-situ measurements relative to the remotely-sensed data set is somewhat arbitrary. We chose a weighting for each in-situ one-hour average measurement that is ten times the total weight of each LOS observation. Since there are on average approximately 600 LOS $g$-level observations used during the 27-day CR periods in 2011 (thus approximately 20 lines of sight per day), the in-situ weighting dominates that of the remote-sensing value total by slightly over a factor of 10 . This weighting for the in-situ data measurement ensures that in-situ densities are accommodated in the analysis as well as the reconstruction resolutions can provide. However, this arbitrarily high weighting leaves little ability for the least squares fit to depart from the in-situ density values which may themselves have significant error. For our forecast analysis, we attempt to match the ACE Level-0 density data as well as possible (even though, as noted, these data may have systematic error with a mean value somewhat lower than other in-situ spacecraft densities measured near the $\mathrm{L}_{1}$ Lagrangian point). The effect of the inclusion of in-situ density or this weighting into the time-dependent tomography leaves the remotely-sensed IPS result beyond the immediate vicinity of Earth relatively unchanged as shown for velocity in Paper 1.

The ACE Level-0 density data generally appear to have a lower mean base value than other in-situ data sets near Earth, or for that matter than the more-refined ACE Level-2 data. For IPS $g$-level values, this presents a problem since a value of $g=1.0$ is supposed to represent a mean ambient solar-wind value. To match densities that have a lower than normal mean requires that a mean $g$-level fit to abnormally low in-situ densities would drive some volumetric density excursions non-physically below zero. To counter this for these ACE Level-0 density data, all scintillation levels below $g=1.0$ are scaled by a factor of the ratio of the mean value determined from the ACE data during the interval relative to an assumed mean level of $5.0 \mathrm{~Np}$. The new mean $g$-level is subtracted from the old $g$-level for those LOS above this value. This somewhat arbitrary remedy may not prove appropriate for all time intervals, but it seems to suffice for the 2011 time interval presented here. 


\section{Comparison of Remote-Sensing and Remote-Sensing Plus In-Situ Inclusion}

An obvious application for incorporating in-situ measurements into the 3D reconstructions is for space-weather forecasting. When the analysis matches observed in-situ values during times when such measurements exist, as demonstrated for velocities in Paper 1, it is reasonable to expect that the density forecast values continuing past that time become more accurate. We compare a sample forecast analysis on 11 November 2011 using time-dependent reconstructions with IPS observations, both without and with incorporating ACE Level-0 data. At this time there was the aftermath of a halo CME observed in the Large Angle and Spectrometric COronagraph (LASCO) C2 and C3 instruments (Brueckner et al., 1995) onboard SOHO, and in STEREO COR 2 coronagraphs (Howard et al., 2008) which erupted from the Sun and was first seen in the LASCO C2 coronagraph on 09 November 2011 at 13:36 UT (Schenk, 2011). Figures 2 and 3 show this CME as in coronagraph observations, and later in the IPS 3D reconstructions as presented on the UCSD website on 11 November. These images have a larger format than those on the UCSD website and slightly different scales in order to better show the events observed at this time. The CME, or rather the CME sequence, heads somewhat to the east of the Sun-Earth line. Here, a slightly earlier CME moves outward to the southeast and more towards the Earth, while a more dominant CME in the LASCO C2 field of view travels rapidly to the solar northeast and is more distant from the Earth. STEREO-B (the behind Earth orbit spacecraft) observes a density enhancement that peaks at about $10 \mathrm{~Np}$ at 18:00 UT on 11 November and another at about 06 UT on 12 November. The ACE Level-0 data show very little change in density at this time (but the Level-0 data are intermittent). The Wind spacecraft data is also absent during this time. From CELIAS there is evidence of a small shock that we assume is associated with the halo $\mathrm{CME}$ at $\sim$ 06:00 UT on 12 November with an associated increase in density of a few Np.

Figure 4 gives a density time series and its correlation for both cases. For comparison with the in-situ data at resolutions commensurate with the 3D reconstructions, the ACE data have been "boxcar averaged" using a 3/4-day-interval filter. The correlation with in-situ density without the inclusion of in-situ measurements during this particular CR is relatively poor; often higher correlations for specific periods near CMEs and/or other whole CRs are reported (e.g., Jackson et al., 2003; Dunn et al., 2005; Bisi et al., 2009a, 2009b) using STELab and other data sets in the reconstructions. As expected, there is a higher correlation (from 0.243 to 0.533 ) with the inclusion of the ACE in-situ density measurements for our reconstructions (Figure 4c, 4d), and this is a significant improvement in the results shown in this example. Far higher correlations $(\geq 0.7)$ over intervals of a single CR are typically found on the UCSD website (see http://ips.ucsd.edu, under Time-Dependent, Correlations 5-day aftercasts). Although this is not as dramatic an improvement as seen in the velocity data, which changes from a non-existent correlation of -0.027 to 0.836 over this interval, at this time of the year there are very few IPS velocity sources available (only a few per day). This is undoubtedly the main reason for the poor correlation using IPS remotely-sensed velocities alone. The better improvement for velocity than for density is probably due to the much smaller excursions from the mean present in the velocity $3 \mathrm{D}$ reconstructions at this time.

\section{Real Time Density Forecasts}

Since early May 2011 (CR2010) we have been operating our time-dependent tomography routine daily at UCSD and forecasting time series values of density and velocity $\sim$ three 


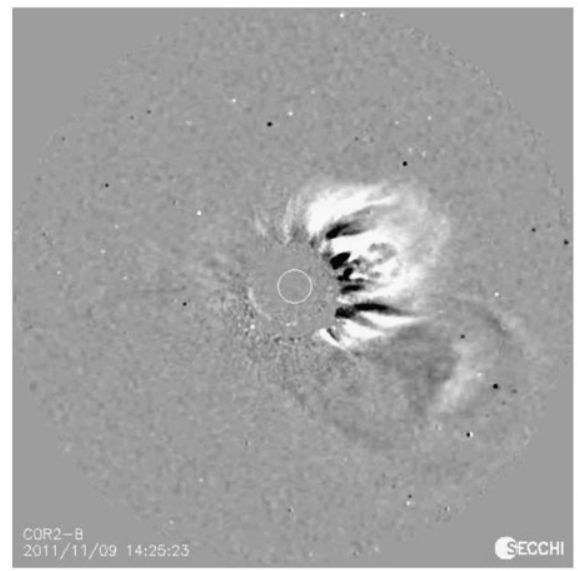

(a)
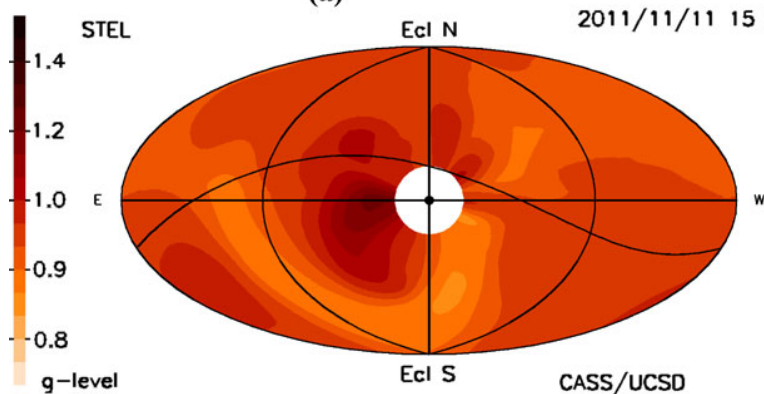

(c)

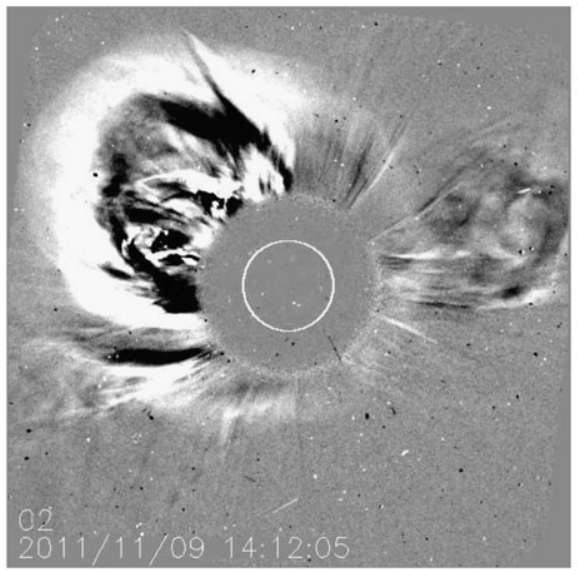

(b)

$2011 / 11 / 1115$ UT

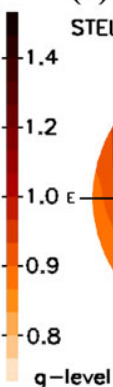

EcI N
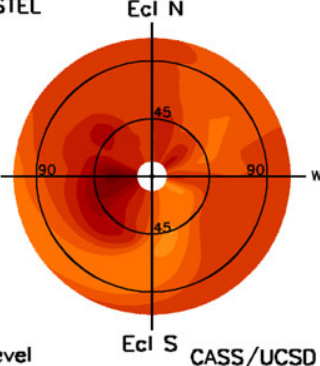

(d)

Figure 2 Coronagraph images and IPS 3D reconstruction extraction 2D images of the 09 November 2011 CMEs. (a) STEREO COR 2 difference image of the CME sequence at 14:25 UT 09 November. STEREO $\mathrm{B}$ is situated $99^{\circ}$ east of the Sun-Earth line on this date. (b) LASCO C2 coronagraph image of the CME sequence at 14:12 UT 09 November. (c) An IPS Hammer-Aitoff $g$-level 3D reconstruction 2D presentation of the CME event sequence at 15:00 UT on 11 November. In this presentation, the Sun is centered and the ecliptic is a horizontal straight line. The outer edge of the image is the single point behind the observer. The IPS $g$-level presentation shown is as used to match modeled IPS $g$-levels from the 3D density values to individual IPS source observations (see Section 2). This presentation gives a measurement of the CME response relative to the mean, and approximately mimics the coronagraph vignetting that is present showing structures more distant from the Sun as brighter than they otherwise would be in the coronagraph image presentations. (d) A similar IPS “fisheye" 3D reconstruction 2D presentation of the CME event sequence at the same time and with the same orientation as (c). The CME sequence is observed to the northeast and southeast, and has reached to about $85^{\circ}$ elongation in the ecliptic, but somewhat beyond that to the northeast. The Sun is centered with elongation circles at $45^{\circ}$ and $90^{\circ}$ indicated in the image. The same $g$-level presentation scale is used as in the Hammer-Aitoff image of (c).

days into the future, and the study given below describes and shows how well we do in practice from the IPS arrays in Japan. In this case the real-time forecasts from the websitesaved files check how well the system performs over an extended time period.

The radio arrays of the STELab system, and in particular for observing IPS $g$-level at Toyokawa, Japan, can only view radio sources crossing the local meridian; the last radio sources observed in Japan are observed at approximately 18:00 h local time, or about 06:00 UT. Data from the day's analysis are downloaded from the STELab arrays, processed automatically at STELab (usually by about 04:00 h local time), and left on a STELab ftp website for public use. An hourly query of this website from UCSD approximately 10 hours 


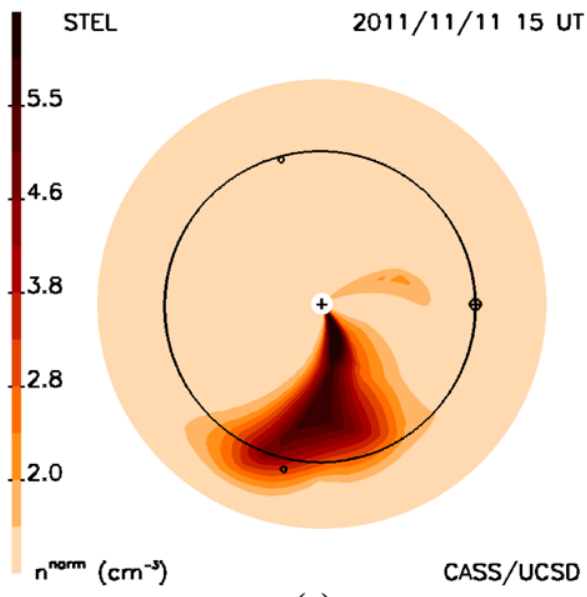

(a)

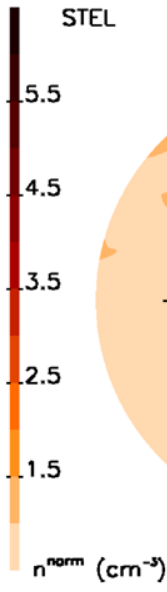

$\mathrm{n}^{\text {norm }}\left(\mathrm{cm}^{-3}\right)$
$2011 / 11 / 1115$ UT

(b)

Figure 3 Density cut displays of the 3D reconstruction of the 09 November 2011 CMEs. The Sun is centered in these plots with the Earth marked $(\oplus)$ on its orbit that is indicated by the circle or line in the plots. (a) An IPS ecliptic cut 3D reconstruction presentation of the CME event sequence at 15:00 UT on 11 November. An $r^{-2}$ correction has been applied to the density in order to maintain approximately the same presentation of features as they move outward from the Sun. The locations of STEREO-A (ahead spacecraft - top) and STEREO-B (bottom) are marked. (b) An IPS meridional cut 3D presentation of the CME event sequence at 15:00 UT on 11 November. Again, an $r^{-2}$ density correction has been applied to the presentation. A small density enhancement can be observed along the Sun-Earth line associated with the oncoming halo CME, but far more material is observed moving outward to the east of the Sun-Earth line.

after the day's data are obtained in Japan (16:00 UT) generally indicates a new file with analyzed data are available at STELab, and these are then downloaded beginning a series of programming steps. For the analysis that includes the real-time in-situ data, this requires that an additional program be run which queries the National Oceanic and Atmospheric Administration (NOAA) computer every hour for real-time ACE data.

It takes approximately 6 minutes to complete the IPS tomography program forecast and to output the associated volumetric files. Once the tomography analysis completes, a further procedure provides visualizations for the website. First, images are made from the current tomography analysis that are presented immediately (within 18 minutes) on the website from the latest tomography run. Following are animations of the original tomographic analysis for use throughout the daily period before the next tomography run is available. The image data sets require updates that change time markers and updated in-situ measurements. These are refreshed hourly from the earlier-run tomography until there are no longer forecast volumetric files resident on the computer.

To evaluate this work, and unlike the analysis shown in Paper 1, we provide a correlation measurement of the forecast change that is presented on our website just following the tomographic run from the actual change observed in ACE data one, two, and three days later and show the first two of these examples in Figure 5. Both the velocity and the density in-situ measurements have been included in the analysis on the website since spring 2011 and this too is different from the Paper 1 analysis that was presented using only the velocity in-situ inclusion. These time series comparisons and correlations give one metric of how well we forecast changes to the in-situ values, and are different from the values available on 


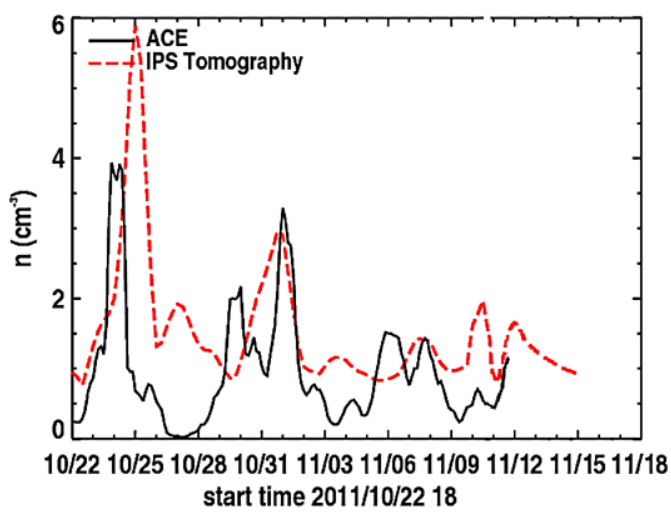

(a)

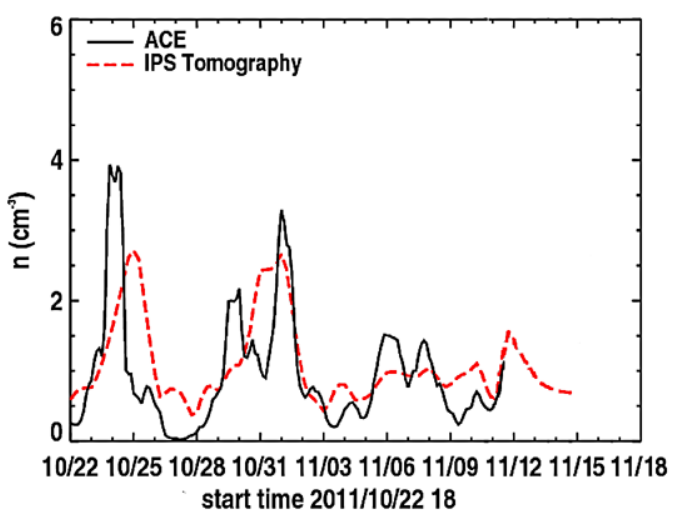

(c) correlation 0.236

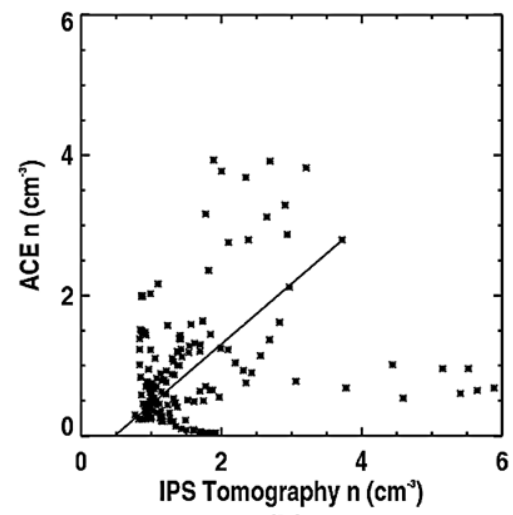

(b)

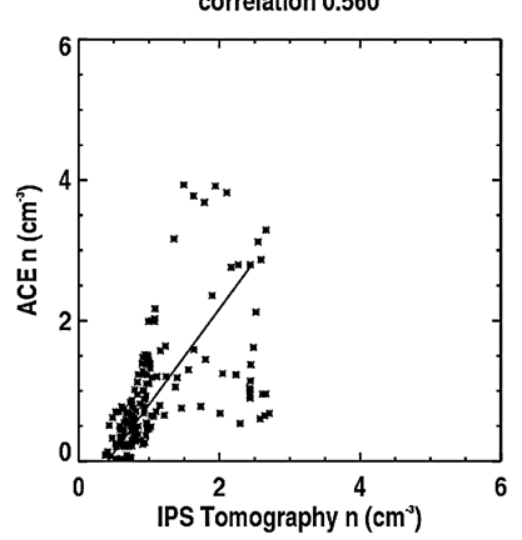

(d)

Figure 4 Density time series extraction from the time-dependent 3D reconstruction using STELab IPS data during the 11 November 2011 event period compared with ACE SWEPAM Level-0 3/4-day averaged data (as explained in the text). The solid-line time series in each plot is the reconstruction result, and the dashed line corresponds to the in-situ measurements from ACE. These ACE data stop at 16:00 UT on 11 November, and continue as forecast in the tomography for another 3 days (dashed line only). (a) and (b) density time series and its correlation without including in-situ data in the reconstruction. (c) and (d) the same, but with the in-situ measurements incorporated.

the UCSD webpage for over half a decade since this current analysis shows the change from the available value. In general in this type of forecast one would like to do better than a value of no change (assuming persistence or that the same value can be extended into the future) from the previous observations in order to claim a success. As can be seen from the example shown in Figure 5 the value forecast is better than assuming no change in the record one and two days in advance. Table 1 gives this same analysis for the six CRs since early May 2011 that we have been operating our website with this new forecast density analysis in place. The CR for the study is given in the first column. Difference correlations 24, 48, and 72 hours in advance of the tomographic run time are shown in the last three columns that are 

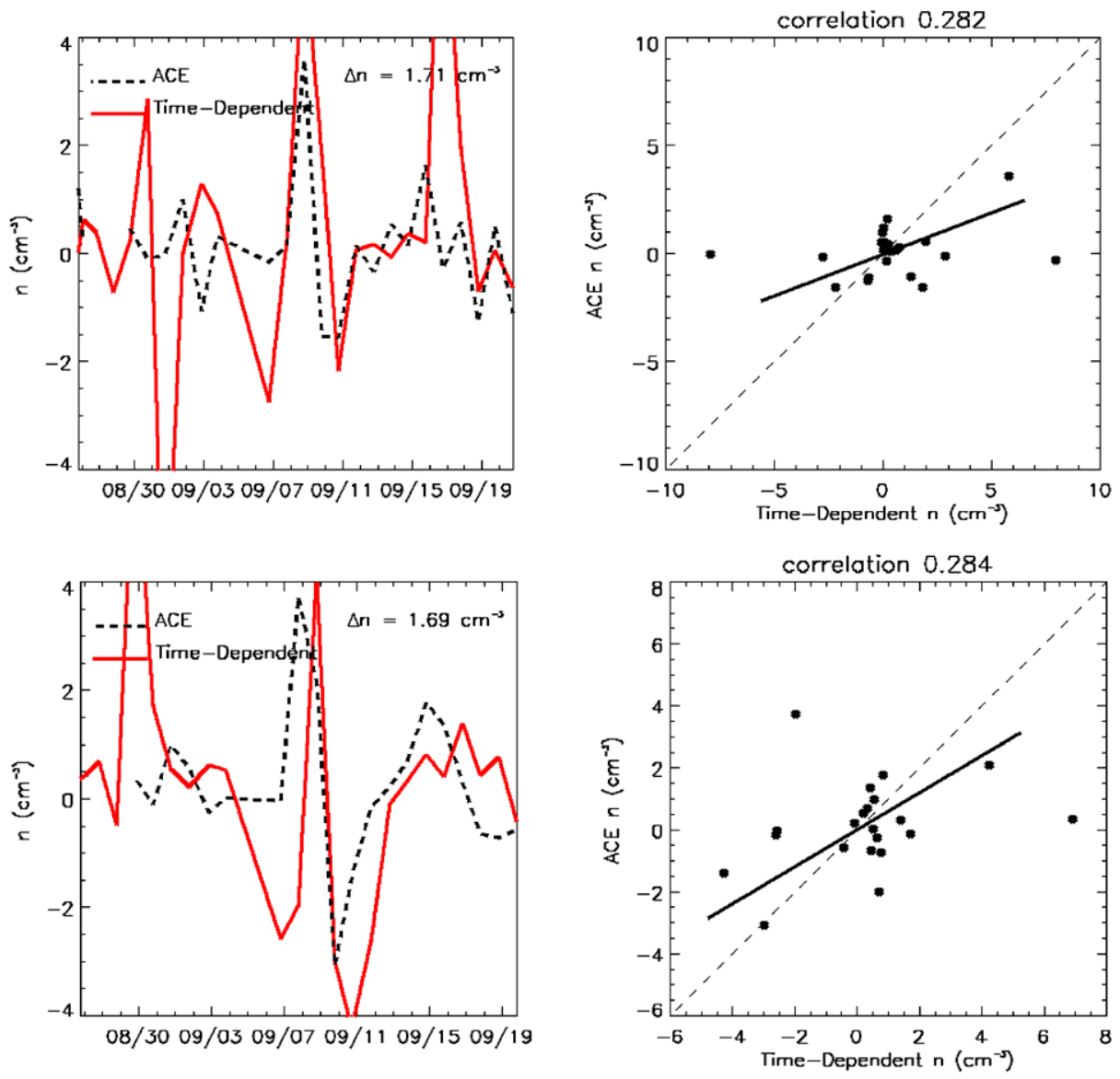

Figure 5 Sample time series and correlation plots for density forecast changes including both STELab IPS and ACE SWEPAM Level-0 data as input for CR2114. ACE density differences from the tomographic run time are shown as the dotted line and on the correlation ordinate, and compared with the tomographic forecast change (solid line) and on the correlation abscissa. A least squares line through the axis origin is drawn in the plots. (a) Change 24 hours following the tomographic run time. (b) Change 48 hours following the tomographic run time.

split into two groups and include the values from the study shown in Figure 5. The density correlations are highlighted and generally show significant positive correlations (or a better than persistence measurement) one day into the future. Two days into the future, the record becomes less positive, and at three days into the future, the record is shown to be even less well forecast. Velocity correlations are also given for completeness in Table 1.

Important in the correlation is not only how well we forecast the future measurement change, but also the size of the future change. This change is not indicated by the correlation, and in a few instances such as the density change for CR2010 and the velocity change for CR2011, the amount of change present in the ACE data are less than $1.0 \mathrm{~Np} \mathrm{~cm}^{-3}$ and $100 \mathrm{~km} \mathrm{~s}^{-1}$, respectively. It is also clear that this type of correlation is only one metric that can be used. We summarize these results further in the final section, and describe how we expect to better these forecasts in the future. 
Table 1 Correlation of IPS change from the change measured by ACE SWEPAM.

\begin{tabular}{|c|c|c|c|c|c|c|}
\hline \multirow{2}{*}{$\begin{array}{l}\text { Carrington } \\
\text { rotation }\end{array}$} & \multicolumn{2}{|c|}{ 24-hour forecast } & \multicolumn{2}{|c|}{ 48-hour forecast } & \multicolumn{2}{|c|}{ 72-hour forecast } \\
\hline & Velocity & Density & Velocity & Density & Velocity & Density \\
\hline 2110 & 0.216 & -0.018 & -0.200 & 0.263 & 0.100 & 0.050 \\
\hline 2111 & -0.004 & 0.364 & -0.010 & -0.049 & -0.374 & 0.300 \\
\hline 2112 & 0.265 & 0.059 & 0.209 & -0.095 & -0.280 & 0.089 \\
\hline 2113 & 0.102 & 0.013 & 0.324 & -0.124 & 0.212 & 0.013 \\
\hline 2114 & 0.356 & 0.282 & 0.268 & 0.284 & -0.126 & -0.347 \\
\hline $2115^{*}$ & 0.290 & 0.320 & 0.150 & 0.280 & -0.082 & 0.145 \\
\hline 2116 & 0.027 & 0.118 & -0.228 & 0.076 & 0.366 & -0.297 \\
\hline
\end{tabular}

* Complete CR analysis unavailable.

\section{Summary and Conclusions}

The inclusion of in-situ measurements into the remote-sensing observations provides a better constraint on the iterated source surface solution, and by outward extension into the heliosphere, provides a positive way of extending current observations into the future as is indicated in the forecast analyses in Figure 5 and Table 1. Of course, an essential test of the $3 \mathrm{D}$ reconstruction technique requires that results incorporating the heavily weighted in-situ data are better fit when in-situ densities are incorporated and this is certified by this study. The in-situ input to the 3D reconstruction program is incorporated in the form of hourly averages, and the output of the reconstruction program is averaged spatially and temporally with the Gaussian filters noted in Section 2. For comparisons commensurate with the resolutions imposed by the tomographic analyses, the ACE data have been averaged using a 3/4-day temporal filter. For this reason alone the comparisons are not expected to be exact, but only close to what is measured in situ, and this is also shown in the current study (Figures 4 and 5).

This article shows for space weather forecast analyses that the present methods provide an improvement in the IPS 3D velocity reconstruction at Earth. In-situ data have been the primary measurements available for study of solar-wind plasma parameters near Earth, and are thus the standard available for comparison. Even so, we do not know a priori whether the ACE Level-0 in-situ density measurements are closer to the correct values than those measured remotely, and we simply assume they are, and we try to match them in the future. Other spacecraft near Earth may give better results and allow better density forecasts than ACE, and especially in the use of the mean level density fit to $g$-level that is mentioned in the last paragraph of Section 2. However, which of the other $\mathrm{L}_{1}$ spacecraft in-situ density measurements can be better forecast using this technique is beyond the scope of the current study, and presently their data are not available in real time to enable forecast analysis.

If the inclusion of in-situ measurements into the tomographic result were simply a way to smooth these so that they blend into the result determined by remote sensing alone, we would not expect much improvement in the remote-sensing forecast results beyond the immediate vicinity of the last in-situ measurements. This does not seem to be the case for velocities as shown by Paper 1, and we speculate that the inclusion of the in-situ measurements near the observing point significantly improves each LOS result. It probably does this by refining information close to the observer, where small amounts of noise might seriously alter the result along the whole LOS. The inclusion of the in-situ data can also eliminate source values 
that are erroneous, since it is possible that a few cause large deviations in our modeling and are not otherwise removed by the iterative-fitting criterion. Although a correct $g$-level analysis undoubtedly helps the analysis of velocity and vice versa, the two parameters of $g$-level (the density proxy) and velocity are nearly separable and the tomographic program converges well and approximates the same answer for the remaining parameter whether or not velocities or $g$-levels alone are available. Currently during the winter months in Japan, only $g$-level values are available from the new STELab Toyokawa array that operates yearround, and with no velocities to help in the analysis, the forecasts have worked satisfactorily throughout the 2011-2012 winter season. This operation of the tomographic program was explored more thoroughly by Jackson et al. (1998), using the archival IPS data sets available at that time; a more comprehensive analysis of this using current STELab Toyokawa $g$-level data, and with and without the inclusion of in-situ data is beyond the scope of the current investigation described here.

We would like to provide a better forecast analysis, and while the metric we have chosen to display is encouraging and shows that generally one or perhaps two days in the future we do better than a simple extension of the current non-inclusion results into the future, the time series changes and the forecast correlations shown are not exceptional. This is surely due in part because the correlations for the remotely-sensed density data comparison with the ACE Level-0 data during this period are not very good. Others plan to provide their own metrics for this same-type analysis (e.g., MacNeice et al., 2011), and perhaps a better way to provide a different metric will be found to improve the forecasts, and consequently these analyses for research purposes using the archival data set.

Limits to the IPS density forecasting technique include the assumptions made to extend $g$-level variations to density forecasts. This is a non-linear process even in weak scattering (for $327 \mathrm{MHz}$ IPS observations, this is generally beyond about $11.5^{\circ}$ elongation), and it assumes that a relationship can be found between $g$-level determinations and density that is the same for all heliospheric structures (see Section 2). For instance, the possibility has often been expressed that the regions behind shocks could have fundamentally more turbulence than the increased density would indicate (e.g., following co-rotating structures - Ananthakrishnan, Coles, and Kaufman, 1980). This has not been checked systematically in these analyses, but there is not much evidence for this so far in the forecast CME shock analyses. The 09 November 2011 halo CME is a good example of an event that provides a shock observed at Earth, and yet has no discernable additional turbulence that provides a higher than expected density. Other innovations are possible in the near future. The assumption is not optimal that all source sizes used in the IPS weighting are of the same value (see Bisi et al., $2010 \mathrm{~b}$ for how source size affects the LOS weighting). Inclusion of measured source sizes (Manoharan and Ananthakrishnan, 1990), or using these same iterative techniques to modify source sizes to provide better $3 \mathrm{D}$ reconstruction fits, could in turn improve tomographic analyses both for research studies and forecasting.

Reducing other limits at STELab could provide forecasts with shorter latency. The actual analysis time required to process the IPS data generally amounts to about three hours each day. The 3D reconstruction analysis uses in-situ data available within an hour of the tomographic run. However, the remotely-sensed IPS observations are at least 10 hours old. Although this data latency could be shortened at STELab, it would require processing the IPS data before local midnight in Japan, and foregoing calibrations of the arrays following data recording. Thus, processing the IPS data to provide updates as sources are observed would help, but is not currently planned, because it is very labor intensive for an instrument where archival research is the primary focus rather than real-time operation.

There are also several limits to the technique to provide a better IPS density forecast that can be overcome by improved analysis systems. Primary limits to the IPS observations are 
the few radio sources that can be recorded by the current STELab system and the unavoidable daily cadence. The first limits the amount of sky coverage and 3D resolution, while the latter means that the fastest outward-moving events can go unseen before they arrive at Earth. These are well-known limits to a single IPS system, and while provision of more lines of sight can be made by using a larger array as at Ooty, India (e.g., Manoharan, 2010), or at Pushchino, Russia (e.g., Chashei et al., 2011), this enhancement quickly becomes an arduous undertaking, especially in Japan where buying parcels of land suitable for large radio arrays adds considerably to the cost of a new or larger array. While an IPS array capable of obtaining over 150,000 lines of sight daily as from the SMEI imager analyses (see Jackson et al., 2010b) is probably not possible, a more modest ground-based venture would still prove to be far less expensive than a spacecraft mission, and could achieve somewhat similar results. The second limit, providing more frequent than once-a-day observations, has a solution by obtaining data for a forecast from different longitudes around the globe and integrating them into a single analysis. However, as yet no other existing IPS radio array operates in real time as does the system at STELab. While there has been an incentive to provide combined analysis using data from different world radio arrays using archival IPS data sets, there has so far been no combined forecast analysis attempted. Building, and especially provisioning, similar radio arrays around the world at different longitude locations is a far more expensive option than using the existing radio arrays. Finally, there are interference problems, some man-made and others caused by Earth-based weather, at the existing single sites and systems. In the past when all similar STELab radio arrays were used to determine scintillation level, the $g$-level determination could be mitigated by selecting the radio site that showed the lowest scintillation level for a given source on that day. The correlations shown in Figure 5 and Table 1 do not provide any means for this assessment, but they could, and it is expected that a quality criterion from each data set would substantially allow poor data to be monitored and flagged as suspect. For instance, a more careful look at the record shows that during CR2112 and CR2113 (July-August 2011) there were many several-day outages caused by lightning from thunderstorms and high-speed wind in part associated with the several typhoons that passed through central Japan during this period. Although such weather-related interference can be mitigated to some extent by careful source editing, there is no real recovery from data outages caused by the inability to operate the radio arrays during and following extreme weather events. For the entries in Table 1, the low-density correlation forecast in July and August is most-certainly affected by these observational outages.

In summary, we show a system that works reasonably well year round to determine the direction and propagation of heliospheric transient phenomena, and to forecast the arrival (or non-arrival) of these structures in the vicinity of the Earth when compared with simply assuming tomorrow's measurements will be the same as today's. We expect that these analyses can still be improved considerably, and that this will lead to better forecasts, and suggest better means of IPS data accrual and editing, and of making the global heliospheric research analysis possible from this unique data set.

Acknowledgements B.V. Jackson, P.P. Hick, M.M. Bisi, J.M. Clover, and A. Buffington were supported at UCSD by NSF grants ATM-0852246, and AGS-1053766 and NASA grant NNX11AB50G. In addition for these analyses, M.M. Bisi was supported by NSF grant ATM-0925023 at UCSD and by a UK STFC Standard Grant to Aberystwyth University, and B.V. Jackson was also supported in part by AFOSR grant 11NE043. The authors wish to acknowledge and thank the group at STELab, Nagoya University not included in the author list (M. Kojima, K. Fujiki, and students) for their continued support, and for making IPS data sets available under the auspices of a joint collaborative agreement between the Center for Astrophysics and Space Sciences at UCSD and STELab, Nagoya University. We also wish to thank Kevin Schenk and the LASCO coronagraph group for the 09 November 2011 halo CME images taken using the C2 coronagraph, and also to the STEREO SECCHI group for making available their COR 2 images. 
Open Access This article is distributed under the terms of the Creative Commons Attribution License which permits any use, distribution, and reproduction in any medium, provided the original author(s) and the source are credited.

\section{References}

Ananthakrishnan, S., Coles, W.A., Kaufman, J.J.: 1980, J. Geophys. Res. 85, 6025.

Asai, K., Kojima, M., Tokumaru, M., Yokobe, A., Jackson, B.V., Hick, P.L., Manoharan, P.K.: 1998, J. Geophys. Res. 103, 1991.

Bisi, M.M., Jackson, B.V., Hick, P.P., Buffington, A., Clover, J.M.: 2007, Adv. Geosci. 14, 161.

Bisi, M.M., Jackson, B.V., Clover, J.M., Manoharan, P.K., Tokumaru, M., Hick, P.P., Buffington, A.: 2009a, Ann. Geophys. 27, 4479.

Bisi, M.M., Jackson, B.V., Buffington, A., Clover, J.M., Hick, P.P., Tokumaru, M.: 2009b, Solar Phys. 256, 201.

Bisi, M.M., Jackson, B.V., Hick, P.P., Buffington, A., Clover, J.M., Tokumaru, M., Fujiki, K.: 2010a, Astrophys. J. Lett. 715, L104.

Bisi, M.M., Jackson, B.V., Breen, A.R., Dorrian, G.D., Fallows, R.A., Clover, J.M., Hick, P.P.: 2010b, Solar Phys. 265, 233.

Brueckner, G.E., Howard, R.A., Koomen, M.J., Korendyke, C.M., Michels, D.J., Moses, J.D., Socker, D.G., Dere, K.P., Lamy, P.L., Llebaria, A., Bout, M.V., Schwenn, R., Simnett, G.M., Bedford, D.K., Eyles, C.J.: 1995, Solar Phys. 162, 357.

Chashei, I.V., Shishov, V.I., Tyul'bashev, S.A., Oreshko, V.V.: 2011, Asia Oceania Geosciences Society Meeting Presentation, Taipei, 8 - 12 August, 2011, p. 270.

Dunn, T., Jackson, B.V., Hick, P.P., Buffington, A., Zhao, X.P.: 2005, Solar Phys. 227, 339.

Domingo, V., Fleck, B., Poland, A.I.: 1995, Space Sci. Rev. 72, 81.

Hewish, A., Scott, P.F., Wills, D.: 1964, Nature 203, 1214.

Hick, P.P., Jackson, B.V.: 2004, Proc. SPIE 5171, 287.

Hovestadt, D., et al.: 1995, Solar Phys. 162, 441.

Howard, R.A., Moses, J.D., Vourlidas, A., Newmark, J.S., Socker, D.G., Plunkett, S.P., Korendyke, C.M., Cook, J.W., Hurley, A., Davila, J.M., Thompson, W.T., St. Cyr, O.C., Mentzell, E., Mehalick, K., Lemen, J.R., Wuelser, J.P., Duncan, D.W., Tarbell, T.D., Wolfson, C.J., Moore, A., Harrison, R.A., Waltham, N.R., Lang, J., Davister, C., Eyles, C.J., Mapson-Menard, H., Simnett, G.M., Halain, J.P., Defise, J.M., Mazy, E., Rochus, P., Mercier, R., Ravet, M.F., Delmotte, F., Auchere, F., Delaboudinière, J.-P., Bothmer, V., Deutsch, W., Wang, D., Richel, N., Cooper, S., Stephens, V., Maahs, G., Baugh, R., Mcmullin, D., Carter, T.: 2008, Space Sci. Rev. 136, 67.

Jackson, B.V., Hick, P.P.: 2005, In: Gary, D.G., Keller, C.U. (eds.) Solar and Space Weather Radiophysics Current Status and Future Developments, ASSL 314, Springer, Berlin, 355.

Jackson, B.V., Hick, P.P., Buffington, A.: 2002, Proc. SPIE 4853, 23.

Jackson, B.V., Hick, P.L., Kojima, M., Yokobe, A.: 1998, J. Geophys. Res. 103, 12049.

Jackson, B.V., Hick, P.P., Buffington, A., Kojima, M., Tokumaru, M., Fujiki, K., Ohmi, T., Yamashita, M.: 2003, In: Velli, M., Bruno, R., Malara, F. (eds.) Proc. Solar Wind X, AIP Conf. Proc. 679, 75.

Jackson, B.V., Buffington, A., Hick, P.P., Wang, X., Webb, D.: 2006, J. Geophys. Res. 111, A04S91.

Jackson, B.V., Boyer, J.A., Hick, P.P., Buffington, A., Bisi, M.M., Crider, D.H.: 2007, Solar Phys. $241,385$.

Jackson, B.V., Bisi, M.M., Hick, P.P., Buffington, A., Clover, J.M., Sun, W.: 2008, J. Geophys. Res. 113, A00A15.

Jackson, B.V., Hick, P.P., Bisi, M.M., Clover, J.M., Buffington, A.: 2010a, Solar Phys. 265, 245.

Jackson, B.V., Buffington, A., Hick, P.P., Bisi, M.M., Clover, J.M.: 2010b, Solar Phys. 265, 257.

Jackson, B.V., Buffington, A., Hick, P.P., Clover, J.M., Bisi, M.M., Webb, D.F.: 2010c, Astrophys. J. 724, 829.

Jackson, B.V., Hick, P.P., Buffington, A., Bisi, M.M., Clover, J.M., Tokumaru, M.: 2010d, Adv. Geosci. 21, 339.

Jackson, B.V., Hamilton, M.S., Hick, P.P., Buffington, A., Bisi, M.M., Clover, J.M., Tokumaru, M., Fujiki, K.: 2011, J. Atmos. Solar-Terr. Phys. 73, 1317. doi:10.1016/j.jastp.2010.11.023.

Kaiser, M.L., Kucera, T.A., Davila, J.M., St. Cyr, O.C., Guhathakurta, M., Christian, E.: 2008, Space Sci. Rev. 136, 5 .

Kojima, M., Kakinuma, T.: 1987, J. Geophys. Res. 92, 7269.

Kojima, M., Tokumaru, M., Watanabe, H., Yokobe, A., Asai, K., Jackson, B.V., Hick, P.L.: 1998, J. Geophys. Res. 103, 1981.

Manoharan, P.K.: 2010, Solar Phys. 265, 137. 
Manoharan, P.K., Ananthakrishnan, S.: 1990, Mon. Not. Roy. Astron. Soc. 244, 691.

MacNeice, P., Taktakishvili, A., Jackson, B., Clover, J., Bisi, M., Arge, N., Odstrcil, D.: 2011, In: AGU Fall Meeting, SH_20,San Francisco, 5-9 December 2011.

McComas, D.J., Bame, S.J., Barker, P., Feldman, W.C., Phillips, J.L., Riley, P., Griffee, J.W.: 1998, Space Sci. Rev. 86, 563 .

Ogilvie, K.W., Desch, M.D.: 1997, Adv. Space Res. 20(445), 559.

Schenk, K.: 2011, 10 November Soho-halo-alert, 111109.

Stone, E.C., Frandsen, A.M., Mewaldt, R.A., Christian, E.R., Margolies, D., Ormes, J.F., Snow, F.: 1998, Space Sci. Rev. 86, 1.

Tokumaru, M., Kojima, M., Fujiki, K., Yamashita, M., Jackson, B.V.: 2005, In: Proceedings of the URSI GA J05-P2, 0193.

Tokumaru, M., Kojima, M., Fujiki, K., Yamashita, M., Jackson, B.V.: 2007, J. Geophys. Res. 112, A05106.

Tokumaru, M., Kojima, M., Fujiki, K., Maruyama, K., Maruyama, Y., Ito, H., Iju, T.: 2011, Radio Sci. 46, RSOF02.

Young, A.T.: 1971, Astrophys. J. 168, 543. 